\title{
Void-mediated formation of Sn quantum dots in a Si matrix
}

\author{
Y. Lei, ${ }^{a}{ }^{\text {P }}$ P. Möck, ${ }^{\text {b) }}$ T. Topuria, and N. D. Browning \\ Department of Physics, University of Illinois at Chicago, 845 West Taylor Street, Chicago, \\ Illinois 60607-7059

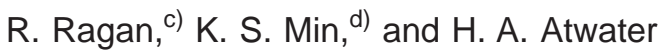 \\ Thomas J. Watson Laboratory of Applied Physics, California Institute of Technology, Pasadena, \\ California 91125
}

(Received 19 February 2003; accepted 21 April 2003)

\begin{abstract}
Atomic scale analysis of Sn quantum dots (QDs) formed during the molecular beam-epitaxy (MBE) growth of $\mathrm{Sn}_{x} \mathrm{Si}_{1-x}(0.05 \leqslant x \leqslant 0.1)$ multilayers in a $\mathrm{Si}$ matrix revealed a void-mediated formation mechanism. Voids below the $\mathrm{Si}$ surface are induced by the lattice mismatch strain between $\mathrm{Sn}_{x} \mathrm{Si}_{1-x}$ layers and $\mathrm{Si}$, taking on their equilibrium tetrakaidecahedron shape. The diffusion of $\mathrm{Sn}$ atoms into these voids leads to an initial rapid coarsening of quantum dots during annealing. Since this formation process is not restricted to $\mathrm{Sn}$, a method to grow QDs may be developed by controlling the formation of voids and the diffusion of materials into these voids during MBE growth. (C) 2003 American Institute of Physics. [DOI: 10.1063/1.1584073]
\end{abstract}

The interest in Sn quantum dots (QDs) embedded in a Si matrix is motivated by their potential applications as band structure engineered direct-band gap elemental semiconductors for optoelectronics and thermophotovoltaics. ${ }^{1}$ As diamond structured $\alpha$-Sn is a direct zero band gap semiconductor, ${ }^{2,3}$ the formation of a substitutional $\mathrm{Sn}_{x} \mathrm{Si}_{1-x}$ alloy is predicted to lead to a direct and tunable energy gap for $x>0.9{ }^{1}$ Quantum confinement is anticipated to increase and thus further tune the energy gap. The growth of ultrathin pseudomorphic $\mathrm{Sn}_{x} \mathrm{Si}_{1-x}$ multilayers with $x=0.05$ and 0.1 by temperature-modulated molecular beam epitaxy (MBE) and $e x$ situ annealing to form Sn-rich QDs has been reported previously. ${ }^{4,5}$ However, the coarsening kinetics during postgrowth annealing revealed an unexpected rapid increase of the average volume of the QDs in the first $2.2 \mathrm{~h}$, though the subsequent growth slowed down to be linear with time. ${ }^{5}$ Typically, any deviation from linear behavior is attributed to diffusion shortcuts related to defects (dislocations) in the material, ${ }^{6}$ but in these samples the QDs nucleated in regions free from dislocations. ${ }^{7}$ In this letter, we report an atomic scale study of $\alpha$-Sn QDs in these materials ${ }^{4,5}$ to elucidate the mechanism behind this initial rapid coarsening, which is of key importance to the overall properties of prospective devices. The implications for other QD systems will also be discussed.

The experiments were performed using a combination of $Z$-contrast imaging and electron energy-loss spectroscopy (EELS) in a $200 \mathrm{kV}$ JEOL 2010F Schottky field emission scanning transmission electron microscope. ${ }^{8}$ This microscope is capable of obtaining essentially incoherent

\footnotetext{
${ }^{a)}$ Electronic mail: ylei1@uic.edu

${ }^{b}$ Present address: Department of Physics, Portland State University, P.O. Box 751, Portland, OR 97207.

${ }^{c}$ Present address: Hewlett-Packard Laboratories, 1501 Page Mill Rd., MS 1123, Palo Alto, CA 94304.

${ }^{d)}$ Present address: Intel Corporation, California Technology and Manufacturing, MS RNB-2-35, 2200 Mission College Blvd., Santa Clara, CA 950528119 .
}

$Z$-contrast images (intensity proportional to atomic number) in the scanning probe mode with a Scherzer resolution of $\sim 0.13 \mathrm{~nm}$. The EELS technique is exploited to quantify fluctuations in chemical composition, as changes in the intensity of core-loss signals give a direct measure of the quantity of specific elements. ${ }^{9}$ Using the $Z$-contrast image as a map to position the probe for spectroscopy allows a direct correlation between the two techniques with atomic spatial resolution. The specimens for the microscopy study were prepared by standard thinning procedures.

Figure 1(a) shows a [110] cross-sectional $Z$-contrast image of the $\mathrm{Sn}_{0.1} \mathrm{Si}_{0.9} / \mathrm{Si}$ sample, indicating QDs with a diameter in the range of 7-10 $\mathrm{nm}$ were formed. Although the majority of the Sn-rich QDs are at the $\mathrm{Sn}_{x} \mathrm{Si}_{1-x}$ layers, there are also QDs within the Si spacer layers, which typically appear to possess a tetrakaidecahedron shape bounded by $\{111\}$ and $\{100\}$ facets [Fig. 1(b)]. The formation of these QDs cannot be explained by the phase separation mechanism ${ }^{5}$ that was proposed to operate within the $\mathrm{Sn}_{x} \mathrm{Si}_{1-x}$ layers. Figure 2(a) shows another QD that has a particularly low intensity, i.e., low Sn content, instead of the essentially uniform brightness presented in Fig. 1(b). This QD also shows higher brightness at the edges, indicating that $\alpha$-Sn lines its interface with the Si matrix. Since it is not
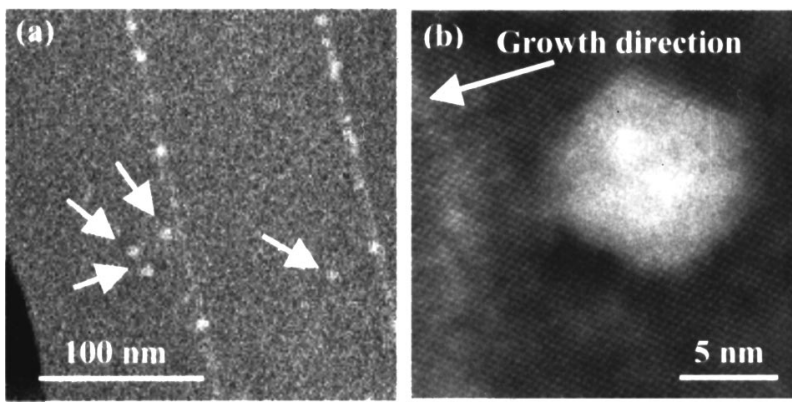

FIG. 1. [110] Cross-sectional Z-contrast images of: (a) the $\mathrm{Sn}_{0.1} \mathrm{Si}_{0.9} / \mathrm{Si}$ multilayer structure and (b) one QD within the Si spacer layers pointed by one of the arrows plotted in (a) 

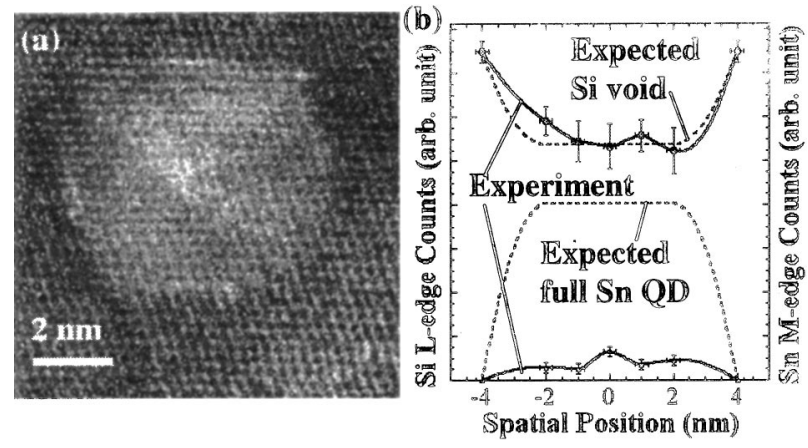

FIG. 2. (a) [110] Cross-sectional Z-contrast image of a QD within the $\mathrm{Si}$ spacer in the $\mathrm{Sn}_{0.1} \mathrm{Si}_{0.9} / \mathrm{Si}$ sample containing a very small amount of $\mathrm{Sn}$. (b) Experimental results of Si $L$-edge counts (solid circles) and $\mathrm{Sn} M$-edge counts (triangles) at the spatial positions along a line across the center of this QD and perpendicular to the growth direction, compared with two theoretical calculations when assuming this QD is an ideal void with no Si inside, and fully filled by $\mathrm{Sn}$, respectively.

viable to determine the $\mathrm{Si}$ content inside the dot from the image intensity alone (as the size of the embedded QD is much smaller than the thickness of the matrix), in order to obtain the compositional information, a series of EELS spectra were acquired along a line across the center of this dot and perpendicular to the [001] growth direction. The Si $L$-edge intensity is plotted as solid circles in Fig. 2(b). The zero point on the spatial position axis corresponds to the position of the center, while the two edge points corresponds to the Si matrix around this QD. The plot indicates a sharp decrease of Si content within the QD relative to the Si matrix, as expected from a QD that contains Sn. However, further EELS analysis of the Sn content reveals that the drop in the $\mathrm{Si}$ concentration is much greater than the increase in the Sn content [the Sn $M$-edge intensity is plotted as triangles in Fig. 2(b)]. In this calculation, Sn atoms were assumed to occupy positions of $\mathrm{Si}$ atoms without lattice expansion, as no obvious lattice expansion was observed from the images. This implies that these QDs are voids in the Si matrix that are only partially filled. This is consistent with the equilibrium shape of a void in $\mathrm{Si}$, which has been shown to be the same tetrakaidecahedron ${ }^{10}$ shape shown in Figs. 1(b) and 2(a).

Two theoretical calculations on an ideal void and a QD fully filled by Sn were further performed and compared with the experimental results. Both this ideal void and QD were assumed to have the same size and shape as the original QD, and to be in a Si matrix of the same thickness in the experiment (this thickness has been calculated from the EELS spectrum). ${ }^{11}$ After the shape parameters of this tetrakaidecahedron were determined based on the image, the effective thickness of $\mathrm{Si}$ along the [110] direction in the region containing this ideal void was calculated. The expected changes in the Si $L$-edge intensity are then plotted in Fig. 2(b), which shows the consistency with the experimental results. Similarly, the expected changes in $\operatorname{Sn} M$-edge intensity in the case of a full Sn QD are also plotted [Fig. 2(b)], and the large discrepancy from the experiment verifies the assertion of a partially occupied Sn structure.

It has been observed that voids of $\sim 10 \mathrm{~nm}$ in diameter and a number density of $\sim 10^{10} \mathrm{~cm}^{-2}$ can form $\sim 10 \mathrm{~nm}$ below a fresh Si surface when it is exposed to air, due to the Downloaded 16 Dec 2005 to 131.215.225.9. Redistribution subject
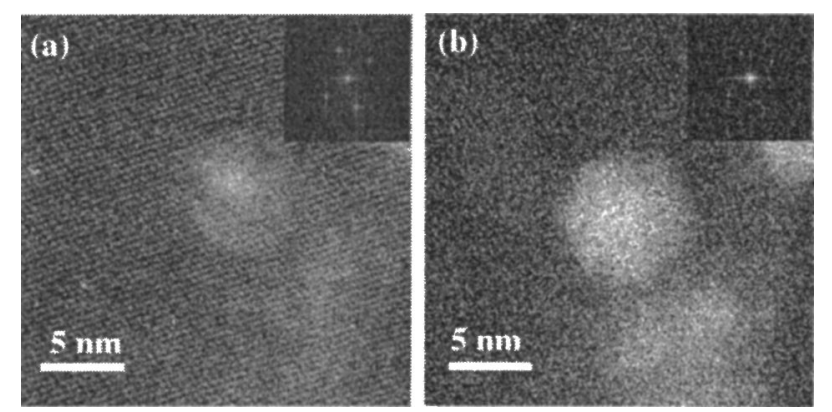

FIG. 3. [110] Cross-sectional Z-contrast images of a QD within the Si spacer layer in the $\mathrm{Sn}_{0.05} \mathrm{Si}_{0.95} / \mathrm{Si}$ sample (a) before and (b) after in situ heating at $300{ }^{\circ} \mathrm{C}$ for $3 \mathrm{~h}$. Fast Fourier transform of the images at the corners indicate the $\mathrm{Sn}$ diamond structure remained same.

compressive strain induced by the growth of $\mathrm{SiO}_{2}$ on the surface. ${ }^{12}$ Thus, the appearance of voids in these multilayer materials is probably induced by the growth of $\mathrm{Sn}_{x} \mathrm{Si}_{1-x}$ layers imposing a similar strain on the $\mathrm{Si}$ surface, and the thermal cycling during the growth ${ }^{4,5}$ finally ensures preformed voids of any shape to grow and reach their equilibrium shape. Therefore, the initial rapid coarsening observed in Ref. 5 may be explained by diffusion shortcuts to these voids.

To confirm this hypothesis, a QD in the Si spacer layer of the $\mathrm{Sn}_{0.05} \mathrm{Si}_{0.95} / \mathrm{Si}$ sample was investigated during an in situ heating process performed at $300^{\circ} \mathrm{C}$ for $3 \mathrm{~h}$. The $Z$-contrast images taken before and after the heating indicate that the intensity within this QD increased (after being normalized according to $\mathrm{Si}$ matrix intensity) and became uniform, while it remained the same tetrakaidecahedron shape (Fig. 3). Although the lattice resolution was lowered a little due to the residual shaking of the heating stage, the fast Fourier transform of the image confirmed the Sn structure as diamond. Since this heating process is exactly analogous to the annealing used in the sample growth, the result is consistent with the QD initially being a void, to which the Sn diffuses during annealing. As the diffusion of Sn atoms into the voids in the Si matrix can further minimize the elastic strain energy between the $\mathrm{Sn}_{x} \mathrm{Si}_{1-x}$ layers and $\mathrm{Si}$ matrix during the phase separation of the $\mathrm{Sn}_{x} \mathrm{Si}_{1-x}$ layers, these voids are the preferential sinks for diffusing Sn atoms. Therefore, this void mediated mechanism dominates at the beginning of QD formation, and results in the initial rapid coarsening.

In conclusion, the analysis of $\mathrm{Sn} \mathrm{QDs}$ in a $\mathrm{Sn}_{x} \mathrm{Si}_{1-x} / \mathrm{Si}$ multilayer system revealed a void mediated formation mechanism. The voids, created by the deposition of lattice mismatched $\mathrm{Sn}_{x} \mathrm{Si}_{1-x}$ layers on the $\mathrm{Si}$, are filled with endotaxially grown $\mathrm{Sn}$ to form pure $\alpha$-Sn QDs. This mechanism explains the initial rapid increase of the size of QDs during postgrowth annealing, and is not restricted to $\mathrm{Sn}$, as any material producing strain on the substrate may induce voids. It is noted that the results here are similar to previous studies that report the formation of hollow and partially filled precipitates in $\mathrm{Si}$ by ion implantation coupled with thermal treatments. ${ }^{13-16}$ However, the size distribution of the precipitates is too wide to be suitable for device quality QD fabrication. By using $\mathrm{MBE}$, the resultant voids have a welldefined shape and narrow size distribution. A method to grow uniform QDs may be developed by controlling the forAlP license or copyright, see http://apl.aip.org/apl/copyright.jsp 
mation of voids and the diffusion of materials into these voids during MBE growth.

This work was supported by NSF under Grant No. DMR-9733895 to N.D.B. and a grant to P.M. by CRB of UIC. The JEOL 2010F was purchased in part with support from NSF under Grant No. DMR-9601792. Nanocrystal synthesis and characterization at Caltech (H.A.A., R.R., and K.S.M.) was supported by NSF under Grant No. ECS0103543.

${ }^{1}$ S. Groves and W. Paul, Phys. Rev. Lett. 11, 194 (1963).

${ }^{2}$ R. A. Soref and C. H. Perry, J. Appl. Phys. 69, 539 (1991).

${ }^{3}$ O. Madelung, in Semconductors: Group IV Elements and III-V Compounds, Data in Science and Technology, edited by R. Poerschke (Springer, Berlin, 1991), p. 22.

${ }^{4}$ K. S. Min and H. A. Atwater, Appl. Phys. Lett. 72, 1884 (1998).

${ }^{5}$ R. Ragan, K. S. Min, and H. A. Atwater, Mater. Sci. Eng., B 87, 204 (2001).

${ }^{6}$ D. A. Porter and K. E. Easterling, Phase Transformations in Metals and
Alloys (Chapman \& Hall, London, New York, 1992), p. 316.

${ }^{7}$ P. Möck, Y. Lei, T. Topuria, N. D. Browning, R. Ragan, K. S. Min, and H. A. Atwater, Proc. SPIE 4807, 71 (2002).

${ }^{8}$ E. M. James and N. D. Browning, Ultramicroscopy 78, 125 (1999).

${ }^{9}$ N. D. Browning, M. F. Chisholm, and S. J. Pennycook, Nature (London) 366, 143 (1993).

${ }^{10}$ D. J. Eaglesham, A. E. White, L. C. Feldman, N. Moriya, and D. C. Jacobson, Phys. Rev. Lett. 70, 1643 (1993).

${ }^{11}$ T. Malis, S. C. Cheng, and R. F. Egerton, J. Electron Microsc. Tech. 8, 193 (1988).

${ }^{12}$ S. Lin, I. Mack, N. Pongkrapan, and P. Fraundorf, SPERE 5, G83 (2002).

${ }^{13}$ A. Meldrum, C. W. White, L. A. Boatner, I. M. Anderson, R. A. Zuhr, E. Sonder, J. D. Budai, and D. O. Henderson, Nucl. Instrum. Methods Phys. Res. B 148, 957 (1999).

${ }^{14}$ A. Meldrum, E. Sonder, R. A. Zuhr, I. M. Anderson, J. D. Budai, C. W. White, L. A. Boatner, and D. O. Henderson, J. Mater. Res. 14, 4489 (1999).

${ }^{15}$ A. Meldrum, S. Honda, C. W. White, R. A. Zuhr, and L. A. Boatner, J. Mater. Res. 16, 2670 (2001).

${ }^{16}$ A. Tchebotareva, J. L. Brebner, S. Roorda, P. Desjardins, and C. W. White, J. Appl. Phys. 92, 4664 (2002). 